\title{
CAMBIOS DE COBERTURA Y FRAGMENTACIÓN DE MANGLARES EN LA REGIÓN DEL SOCONUSCO, CHIAPAS, MÉXICO, 1994-2011
}

\author{
LAND COVERAGE CHANGES AND FRAGMENTATION \\ OF MANGROVES IN THE SOCONUSCO REGION, \\ CHIAPAS, MEXICO, 1994-2011
}

\author{
Emilio I. Romero-Berny ${ }^{1}$ \\ Universidad de Ciencias y Artes de Chiapas \\ Joanna Acosta-Velázquez ${ }^{2}$ \\ El Colegio de la Frontera Sur \\ Cristian Tovilla-Hernández ${ }^{3}$ \\ El Colegio de la Frontera Sur-Unidad Tapachula \\ Birgit Schmook ${ }^{4}$ \\ El Colegio de la Frontera Sur-Unidad Chetumal \\ Rocio Gómez-Ortega ${ }^{5}$ \\ El Colegio de la Frontera Sur
}

1 Maestro en Ciencias en Recursos naturales y Desarrollo rural. Docente. Centro de Investigaciones Costeras, Universidad de Ciencias y Artes de Chiapas. emilio.romero.ex@unicach.mx

2 Maestra en Ciencias (Manejo ambiental). Doctorante. Programa de Posgrado en Ecología y Desarrollo sustentable, El Colegio de la Frontera Sur. joanna.acosta@gmail.com

3 Doctor en Ciencias (Biología). Investigador. Departamento de Ciencias de la Sustentabilidad, El Colegio de la Frontera Sur-Unidad Tapachula. ctovilla@ecosur.mx

4 Doctora en Geografía social. Investigadora. Departamento de Conservación de la Biodiversidad, El Colegio de la Frontera Sur-Unidad Chetumal.bschmook@ecosur.mx

5 Maestra en Ciencias en Recursos naturales y Desarrollo rural. Doctorante. Programa de Posgrado en Ecología y Desarrollo sustentable, El Colegio de la Frontera Sur. rogomez@ecosur.edu.mx 
Emilio I. Romero-Berny, Joanna Acosta-Velázquez, Cristian Tovilla-Hernández,

Birgit Schmook, Rocio Gómez-Ortega. Land coverage changes and fragmentation of

mangroves in the Soconusco Region, Chiapas, Mexico, 1994-2011

\title{
RESUMEN
}

Se analizó la dinámica espacial de la cobertura de manglar en el humedal costero El Cabildo-Amatal-Gancho Murillo, Chiapas, México, determinando su impacto por procesos de fragmentación. El análisis de cambios demostró una pérdida de 509 ha de manglar en un período de 17 años y una tasa de deforestación anual del 1.05\%. Las métricas estimadas indican que el sistema se conforma por 38 parches de manglar mayores a 0.36 ha en el 2011, presentando un aumento del $58 \%$ en su número con respecto a 1994. La dimensión fractal de 1.4 y la tasa de fragmentación indican un paisaje impactado. Los resultados muestran que debido a los cambios en el sistema es necesario implementar planes de manejo costero.

Palabras clave: manglar, percepción remota, SIG, métricas, tasa de deforestación.

\begin{abstract}
We analyzed the spatial dynamics of the mangrove coverage of El Cabildo-Amatal-Gancho Murillo coastal wetland in Chiapas, Mexico by determining their impact by fragmentation processes. The analysis of changes showed a loss of 509 ha of mangrove in a 17 -year period and an annual deforestation rate of $1.05 \%$. The metrics indicate that the system was composed of 38 largest mangrove patches greater than 0.36 ha in 2011 , which represents an increase of $58 \%$ regarding its size in the year 1994. The fractal dimension of 1.4 and the fragmentation rate estimated suggest that the landscape has been impacted. Altogether, the results show that, due to changes in the system, it is necessary to implement coastal management plans.
\end{abstract}

Keywords: mangrove, remote sensing, GIS, metrics, deforestation rate

\section{Introducción}

Los manglares, comunidades vegetales arbóreas o arbustivas asociadas a sistemas costeros, abarcan $137,760 \mathrm{~km}^{2}$ de las costas tropicales y subtropicales en el mundo (Giri et al., 2011). Tienen un papel fundamental en la estabilidad climática, al ser los sumideros de carbono más eficientes en los trópicos; además, de sostener pesquerías y proteger las costas contra fenómenos hidrometeorológicos (Duke et al., 2007).

A pesar de los numerosos servicios ambientales que proporcionan los manglares, se están perdiendo en tasas aceleradas a escala global. Entre 1980 y 2000 se perdió el 35\% de los manglares del mundo (Valiela et al., 2001). En México se estima una extensión de 770,057 ha de manglar (CONABIO, 2009). De acuerdo con FAO (2007), México es uno de los 10 países que presenta una de las tasas de pérdida de manglares más altas del mundo con un 2.1\% anual (1990-2000). Entre los impactos directos de origen antropogénico que afectan a sus manglares, se encuentran, principalmente la deforestación para el desarrollo portuario, urbano, turístico, acuícola y agropecuario, industria petrolera y aprovechamiento forestal. Indirectamente el manglar se ve afectado por azolvamiento, alteración 
hidrológica, hipersalinidad y eutrofización, cuyo origen puede ubicarse en el impacto a otros ecosistemas que componen la cuenca y la zona marina adyacente (Agraz-Hernández y Flores-Verdugo, 2005; Flores-Verdugo, Agraz-Hernández y Benítez-Pardo, 2007).

La deforestación, frecuentemente, implica fragmentación el cual es un proceso complejo que incluye la división del hábitat, e involucra cambios múltiples de pérdida de ecosistemas, aumento en el aislamiento y cambios en la calidad de los parches de vegetación natural. A escala de paisaje afecta la dinámica ecológica, limitando la conectividad y modificando la estructura y composición de las comunidades (Hargis et al., 1998). La fragmentación, es un proceso que pocas veces se ha evaluado en manglares, existiendo un desconocimiento generalizado sobre sus efectos a diferentes escalas espaciales y temporales.

La constante dinámica de cambios en la zona costera y la alta tasa de deforestación de manglares, demandan la aplicación de marcos conceptuales y herramientas que permitan evaluarlos eficientemente y con exactitud a distintas escalas espaciales y temporales. Dadas las complicaciones en el acceso que impone el trabajo de campo en zonas intermareales, la percepción remota y los Sistema de Información Geográfica, son herramientas útiles para dar seguimiento a variables ambientales a diferentes escalas espaciales y temporales como los cambios en el tamaño y forma de los parches de vegetación (Jones y Vaughan, 2010).

El objetivo de este trabajo es determinar los cambios en la cobertura del manglar y su tasa de deforestación para un periodo de 17 años en la región sur del Soconusco, Chiapas, a partir del análisis de imágenes satelitales; y cuantificar métricas de clase asociadas a la fragmentación utilizando un Sistema de Información Geográfica.

\section{Área de estudio}

El Soconusco constituye una región biocultural localizada al sur del estado de Chiapas, México. Sus límites son variables dependiendo el enfoque, histórico, político, productivo o ambiental con el que se aproxime a su estudio. Fisiográficamente, comprende la vertiente del Pacífico de la Sierra Madre de Chiapas y su planicie costera, limitando al norte con la subcuenca del río Novillero y al sur con la del Suchiate en la frontera México-Guatemala (Bassols et al., 1974; Sánchez y Jarquín, 2004). El 
Emilio I. Romero-Berny, Joanna Acosta-Velázquez, Cristian Tovilla-Hernández,

Birgit Schmook, Rocio Gómez-Ortega. Land coverage changes and fragmentation of mangroves in the Soconusco Region, Chiapas, Mexico, 1994-2011

área estudiada, se localiza entre los $14.741-14.533^{\circ} \mathrm{N}$ y $92.43^{\circ}-92.188$ $\mathrm{O}$ y cuenta con una extensión aproximada de 10,244 ha. Prevalece un clima cálido-subhúmedo con un precipitación anual de $1,578.4 \mathrm{~mm}$ y una temperatura promedio de $26.5^{\circ} \mathrm{C}$. Sobresalen sus manglares compuestos por Rhizophora mangle, Laguncularia racemosa, Avicennia germinans y Conocarpus erectus, cuyos rodales pueden alcanzar una altura de $16 \mathrm{~m}$, área basal de $36 \mathrm{~m}^{2} /$ ha y densidad de 4,200 árboles/ha (Tovilla-Hernández y Romero-Berny, 2012).

Figura 1. Área de estudio.

$\mathrm{A}=$ Estado de Chiapas; $\mathrm{B}=$ Región Soconusco

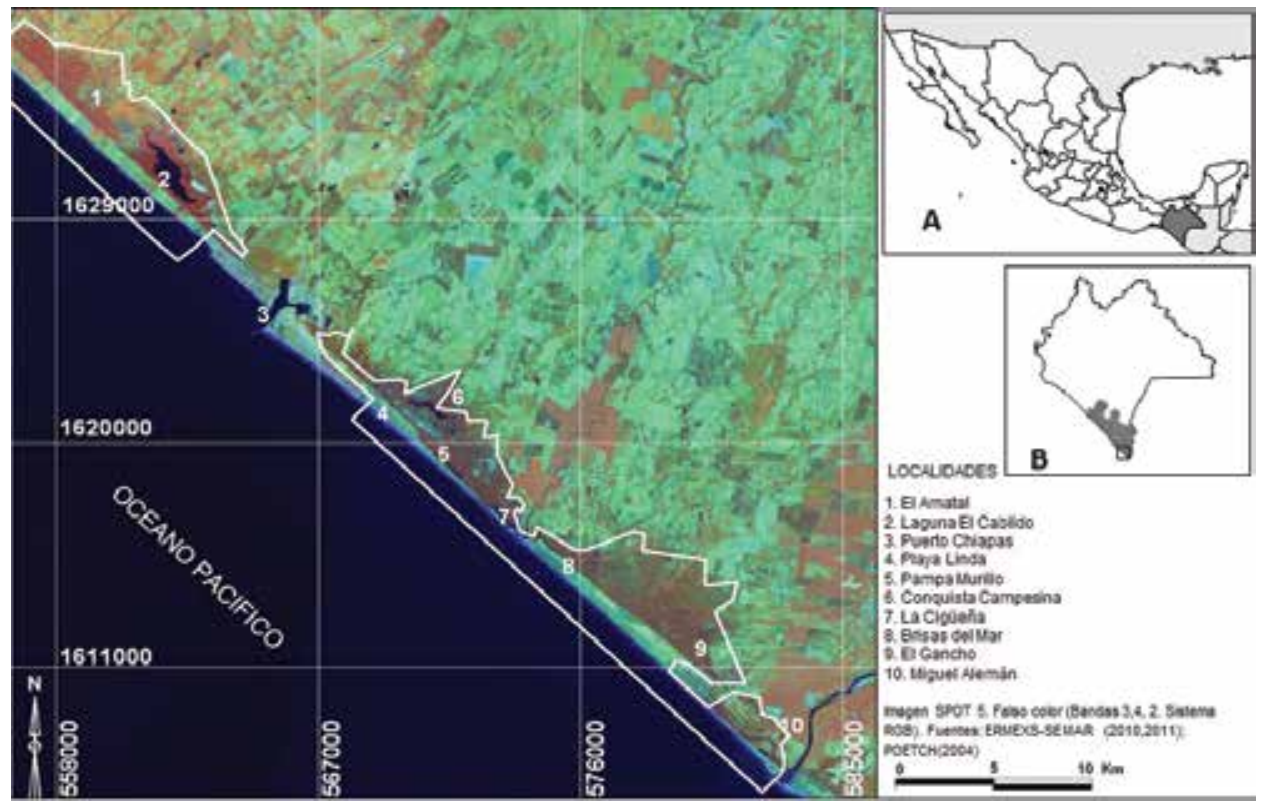

El sistema es de importancia regional para la conservación, presentando las siguientes categorías de manejo: Región Marina Prioritaria No.40, Región Hidrológica Prioritaria No.32, Área de Importancia para la Conservación de Aves No.51, sitio de manglar con relevancia biológica PS27, sitios RAMSAR y Zonas Sujetas a Conservación Ecológica Cabildo-Amatal y El Gancho-Murillo (Arriaga-Cabrera et al., 1998a, 1998b; Ovalle-Estrada y Vásquez-Lule, 2009). En el área de estudio inciden 13 
localidades con una población total de 12,325 habitantes (Gobierno de Chiapas 2010a; 2010b), encontrándose inmersa en una de las regiones de la frontera sur mexicana de mayor dinámica social y económica. Destaca, la producción agropecuaria y la pesca ribereña como principales actividades productivas, así como un acelerado crecimiento urbano debido a una mayor inversión en los sectores industrial y turístico (Sánchez y Jarquín, 2004). La región se ha visto afectada por la influencia de fenómenos hidrometeorológicos, siendo notables por su impacto el huracán "Stan" en 2005 y la tormenta tropical "Bárbara" en 2007, infiriéndose un incremento de la vulnerabilidad regional a causa de políticas de manejo poco adecuadas a nivel de cuenca, que han favorecido la deforestación y la modificación de cauces de ríos y arroyos (figura 1).

\section{Métodos}

Procesamiento de imágenes y mapeo del manglar

Se obtuvieron 4 escenas de los sensores SPOT 5 y LANDSAT TM con las características detalladas en el cuadro 1, las cuales fueron homologadas a una resolución de $30 \mathrm{~m} /$ pixel y recortadas con base en el polígono del sitio de manglar PS27.

Cuadro 1. Sumario de las características de las imágenes satelitales utilizadas en este estudio.

\begin{tabular}{|l|l|l|}
\hline \multirow{2}{*}{\multicolumn{1}{c|}{ Característica }} & \multicolumn{2}{c|}{ Satélite } \\
\cline { 2 - 3 } & \multicolumn{1}{|c|}{ SPOT } & \multicolumn{1}{c|}{ LANDSAT } \\
\hline Sensor & 5 & TM \\
\hline Resolución espectral & 4 bandas & 7 bandas \\
\hline Resolución espacial & $10 \mathrm{~m}$ & $30 \mathrm{~m}$ \\
\hline Clave, fecha de toma & $604-321,30 / 01 / 2011$ & $21 / 50,05 / 01 / 1994$ \\
\hline & $605-321,10 / 01 / 2011$ & \\
\hline & $604-320,10 / 12 / 2010$ & \\
\hline
\end{tabular}

Fuente: ERMEXS-SEMAR, U.S. Geological Survey.

Se realizó un compuesto en falso color con las bandas espectrales 4 , 5, 3 (RGB) para la escena de 1994 y 3, 4, 2 (RGB) para la escena de 2011, y sobre cada una se sobrepuso un mapa temático de la clase "Manglar" generado en el proyecto "Manglares de México" (CONABIO, 2009), actualizando la distribución de la cobertura para 2011, mediante el método de 
Emilio I. Romero-Berny, Joanna Acosta-Velázquez, Cristian Tovilla-Hernández,

Birgit Schmook, Rocio Gómez-Ortega. Land coverage changes and fragmentation of

mangroves in the Soconusco Region, Chiapas, Mexico, 1994-2011

interpretación interdependiente (FAO, 1996). Se utilizó el mismo método en retrospectiva para generar el mapa de 1994, combinando la herramienta de segmentación por regiones de crecimiento espectral, y la interpretación visual con verificación en campo (Díaz-Gallegos y Acosta-Velázquez, 2009). Los mapas se filtraron con ventanas de $3 \times 3$ pixeles, usando la moda como medida de estandarización para reducir las variaciones aleatorias de color y brillo en la imagen.

Para el mapa 2011, la estimación de la exactitud se realizó verificando 145 puntos distribuidos al azar, con el catálogo de fotografías panorámicas del proyecto "Manglares de México" (CONABIO, 2009) y con recorridos de campo. Se elaboró una matriz de error para identificar inconsistencias entre la cobertura verificada y la cobertura generada a partir de la clasificación, con la que se calculó la exactitud global, obtenida a partir de la sumatoria de las unidades correctamente clasificadas dividida entre el número total de unidades de la matriz; la exactitud del productor, calculada dividiendo las unidades correctamente clasificadas en una clase dada, entre el número total de unidades de los datos de referencia para esta clase, esto representa el porcentaje de unidades identificadas en el mapa; y la exactitud del usuario, obtenida de la división de unidades correctamente clasificadas entre el número de unidades. Se estimó el coeficiente de Kappa, para evaluar si la clasificación realizada presentaba una exactitud, significativamente mayor a la que se hubiera logrado con un método al azar (Congalton y Green, 1999). Se utilizó la fórmula

$$
\hat{\mathrm{k}}=\mathrm{n} \sum_{1, \mathrm{n}} \mathrm{Xii}-\sum_{\mathrm{i}=1, \mathrm{n}} \mathrm{Xi}+\mathrm{X}+\mathrm{i} / \mathrm{n}^{2} \sum_{\mathrm{i}=1, \mathrm{~m}} \mathrm{Xi}+\mathrm{X}+\mathrm{i}
$$

en donde ${ }^{\wedge} \mathrm{k}=$ Coeficiente de Kappa, $\mathrm{i}=$ clase, $\mathrm{n}=$ tamaño de muestra, $\mathrm{Xii}=$ elementos clasificados correctamente, $\mathrm{Xi}+\mathrm{X}+\mathrm{i}=$ elementos totales de cada clase.

El pre-procesamiento de imágenes y el procesamiento de escenas se llevaron a cabo con herramientas del software ERDAS ${ }^{\circledR}$ Imagine 9.1 (Leica).

\section{Cambios de cobertura y tasa de deforestación}

El análisis de cambios de cobertura se realizó a partir de la combinación de la clase "Manglar" de las escenas 1994 y 2011 con el módulo 
"Land Change Modeler" del software IDRISI ® Taiga (Clark University).

Se calculó la tasa de deforestación con la siguiente fórmula:

$$
\delta=[\mathrm{S} 2 / \mathrm{S} 1]^{1 / \mathrm{n}}-1
$$

en donde: $\delta=$ tasa de deforestación, $\mathrm{S} 1=$ Superficie en el tiempo 1, $\mathrm{S} 2=$ Superficie en el tiempo 2 y $\mathrm{n}=$ número de años entre ambas fechas (FAO, 1996).

\section{Análisis de fragmentación}

Se vectorizaron las coberturas de la clase "Manglar" de 1994 y 2011, considerándose un tamaño mínimo de parche de 4 pixeles equivalente a 0.36 ha. Se estimaron métricas a nivel de clase con el uso de la herramienta "Spatial Statistics" de la extensión Patch Analyst 3.1 (Elkie et al, 1999) en el software ArcView ® 3.3 (ESRI): Número de parches (NumP), Tamaño medio de parche (MPS), Borde medio de parche (MPE), Índice de Forma (MSI) y Dimensión fractal (MPFD).

Las métricas consideradas se describen en el cuadro 2. Una vez obtenido el número de parches de ambos años, se calculó la tasa de fragmentación a partir de la fórmula siguiente:

$$
\text { Tasa de fragmentación }=[\mathrm{NP} 2 / \mathrm{NP} 1]^{1 / \mathrm{n}}-1
$$

en donde: NP1=Número de parches en el tiempo 1, NP2=Número de parches en el tiempo 2 y n=número de años entre las dos fechas.

Los valores medios de las métricas de 1994 y 2011 se compararon mediante una prueba no paramétrica de U Mann-Whitney, al no cumplirse el supuesto de normalidad en los datos con base en el test Shapiro-Wilks. 
Emilio I. Romero-Berny, Joanna Acosta-Velázquez, Cristian Tovilla-Hernández,

Birgit Schmook, Rocio Gómez-Ortega. Land coverage changes and fragmentation of mangroves in the Soconusco Region, Chiapas, Mexico, 1994-2011

Cuadro 2. Métricas a nivel de clase consideradas en este estudio.

\begin{tabular}{|l|l|}
\hline \multicolumn{1}{|c|}{ Métrica } & \multicolumn{1}{c|}{ Descripción } \\
\hline Numero de parches & Cantidad de parches o fragmentos de una clase. \\
\hline Tamaño medio de parche & El área ( $\mathrm{m}^{2}$ o has.) que ocupa un parche. \\
\hline Borde medio de parche & Media de las longitudes de borde de un parche. \\
\hline Índice de forma & $\begin{array}{l}\text { Relación área-perímetro ajustada a un cuadrado o } \\
\text { círculo (para vectores). Las formas más compactas } \\
\text { tienden a 1 y este valor se incrementa conforme } \\
\text { aumenta la irregularidad de los parches. }\end{array}$ \\
\hline Dimensión fractal & $\begin{array}{l}\text { Medida o índice de la complejidad de la forma } \\
\text { de un parche. Toma valores de 1 a 2. Los valores } \\
\text { más cercanos a 1 indican perímetros regulares y } \\
\text { valores próximos a 2 son mostrados por formas } \\
\text { más complejas. }\end{array}$ \\
\hline
\end{tabular}

\section{Resultados}

\section{Cambios de cobertura}

La exactitud global del mapa temático de la distribución de manglares en 2011 fue de 97\%, con un valor del índice de Kappa igual a 0.94; esto indica, una alta exactitud de clasificación, las de usuario y productor presentaron valores mayores a $95 \%$, considerando alta concordancia entre los datos de referencia y los de la clasificación (Cuadro 3).

Las superficies de manglar estimadas fueron de 3,118 ha en 1994 y 2,609 ha en 2011 (figura 2). La pérdida neta de manglares para un periodo de 17 años se estimó en 509 ha, lo que representa el 16.3\% de su cobertura original en 1994 y presentando una tasa media de deforestación anual de $1.05 \%$, equivalente a 30 ha por año. 
Figura 2. Mapas temáticos de la clase Manglar para 1994 y 2011.
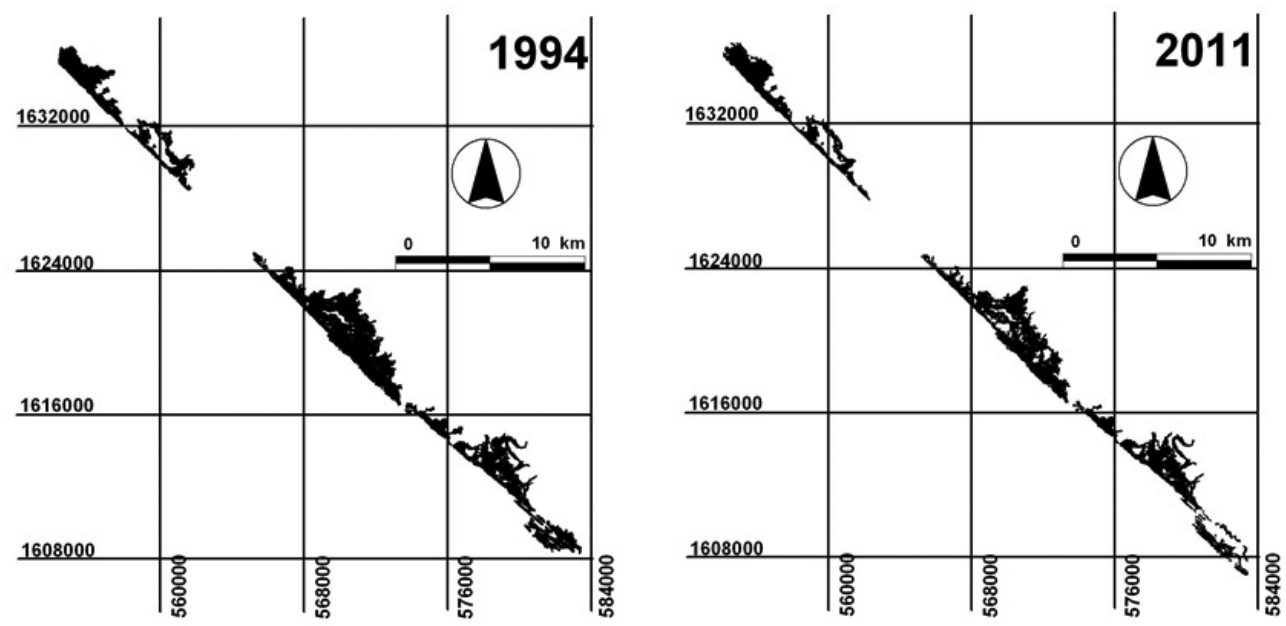

Cuadro 3. Matriz de error de la clasificación para el mapa 2011 y evaluación de la exactitud.

\begin{tabular}{|l|c|c|c|c|}
\hline \multicolumn{1}{|c|}{ Clase } & \multicolumn{3}{|c|}{ Datos de referencia } & $\begin{array}{c}\text { Exactitud de usuario } \\
\text { (\%) }\end{array}$ \\
\hline & Manglar & $\begin{array}{l}\text { Otras } \\
\text { coberturas }\end{array}$ & $\begin{array}{l}\text { Total } \\
\text { columnas }\end{array}$ & \\
\hline Manglar & 71 & 1 & 72 & 98.6 \\
\hline Otras coberturas & 3 & 70 & 73 & 95.9 \\
\hline Total renglones & 74 & 71 & 145 & \\
\hline $\begin{array}{l}\text { Exactitud del } \\
\text { productor (\%) }\end{array}$ & 95.9 & 98.6 & & $\begin{array}{c}\text { Exactitud global (\%) } \\
97\end{array}$ \\
\hline
\end{tabular}

\section{Análisis de fragmentación}

Los valores de las métricas para la clase manglar se presentan en el cuadro 4.

Para 2011, el número de parches (NumP) se incrementó en un 58\% con respecto al número estimado en 1994. La variación en el tamaño medio de parche (MPS), muestra una reducción significativa del área $(W=424$, $p<0.0001$ ), evidenciando la división de parches durante el periodo analizado. Asimismo, el borde medio de parche (MPE), presenta una disminución en este periodo con diferencias significativas ( $W=398, p<0.0001)$. El índice de forma (MSI), muestra que se mantuvo cierta condición de complejidad 
Emilio I. Romero-Berny, Joanna Acosta-Velázquez, Cristian Tovilla-Hernández,

Birgit Schmook, Rocio Gómez-Ortega. Land coverage changes and fragmentation of

mangroves in the Soconusco Region, Chiapas, Mexico, 1994-2011

de los parches, a pesar de una disminución en el valor, no se encontraron diferencias significativas entre ambas fechas $(W=408, p=0.774)$. Un resultado similar se obtuvo en el análisis de la dimensión fractal (MPFD), en donde se confirma la tendencia irregular en los parches sin una variación significativa en el valor $(W=215, p=0.239)$.

La tasa de fragmentación estimada fue de $4.9 \%$ anual para un periodo de 17 años.

Cuadro 4. Valores medios de métricas de paisaje ( \pm desviación estándar) y comparación entre fechas.

\begin{tabular}{|l|l|l|l|}
\hline \multicolumn{1}{|c|}{ Métrica } & \multicolumn{1}{c|}{$\mathbf{1 9 9 4}$} & \multicolumn{1}{c|}{$\mathbf{2 0 1 1}$} & \multicolumn{1}{c|}{ W } \\
\hline NumP & 16 & 38 & \\
\hline MPS & $163.9( \pm 334.8)$ & $63.4( \pm 190.3)$ & $424 * * *$ \\
\hline MPE & $16,424.2( \pm 26,596)$ & $8,777.6( \pm 23,204)$ & $398 * * *$ \\
\hline MPI & $3.3( \pm 2.3)$ & $2.7( \pm 2.0)$ & $408 \mathrm{~ns}$ \\
\hline MPFD & $1.4( \pm 0.0)$ & $1.4( \pm 0.0)$ & $315 \mathrm{~ns}$ \\
\hline
\end{tabular}

Nivel de significancia del test $U$ de Mann-Whitney: ***: $p<0.001$, ns: no significativo.

\section{Discusión de resultados}

El método de clasificación híbrida utilizado en este estudio, permitió delimitar con mayor precisión la cobertura de manglar (Díaz-Gallegos y Acosta-Velázquez, 2009), generándose mapas de alta exactitud. Congalton y Green (1999) consideran que una clasificación aceptable debe exhibir una exactitud global igual o superior a 85\%. Monzalvo (2006) reconoce que los altos niveles de exactitud global (>90\%) son raros en éste tipo de clasificaciones, y el valor obtenido en este estudio se debe a que la clase "Manglar" es aislada del resto de las coberturas por medio de la digitalización de máscaras. Estos resultados permiten asumir que el mapa temático de 2011, representa, adecuadamente, la extensión y distribución de la vegetación de manglar. En los mapas generados no es posible apreciar la interacción de clases, pero a cambio se potencializa la eficacia de clasificación de la cobertura de interés, reduciendo posibles sesgos en el análisis de fragmentación (Altamirano et al., 2012).

En el sur del Soconusco, la tasa de pérdida de manglares es considerada alta, tomando en cuenta que incluye zonas sujetas a conservación. No obstante, los valores variarán dependiendo los agentes de deforestación, la 
región y la temporalidad, por lo que deben considerarse como un elemento parcial de comparación en un análisis multitemporal.

Como referencia, se presentan tasas de pérdida de manglares para áreas del Pacífico mexicano en el cuadro 5. Algunas áreas muestran altas tasas de deforestación, como ocurre en Barra de Navidad, 3.2\% en un periodo de 10 años, en donde el manglar se ha visto afectado por un creciente desarrollo urbano (Holland et al., 2011). En caso opuesto, la menor es reportada para el Parque Nacional Lagunas de Chacahua, con una tasa de deforestación de $0.02 \%$ en un periodo de 21 años, en donde se han reportado áreas de recuperación del manglar, quizá, como resultado de un adecuado manejo del ecosistema en una área protegida federal (Carbajal, 2010).

Además, de la pérdida de cobertura estimada, el aumento en el número de parches y el análisis de métricas, evidencian que el manglar en esta zona se encuentra sujeto a un proceso de fragmentación. Los índices de área y borde total, mostraron tendencias negativas. McGarigal (2002), señala que la reducción progresiva en el área de los parches es un indicador clave del proceso de fragmentación de un hábitat.

Cuadro 5. Tasas de deforestación $(\delta)$ en áreas de manglar del Pacífico de México. ${ }^{1}$

\begin{tabular}{|c|c|c|c|c|}
\hline \multirow[b]{2}{*}{ Sistema/Estado } & \multirow[b]{2}{*}{ Periodo } & \multicolumn{2}{|c|}{ Manglar (ha) } & \\
\hline & & T1 & $\mathbf{T 2}$ & $\delta$ \\
\hline $\begin{array}{l}\text { Bahía Magdalena-Bahía Almejas (Baja } \\
\text { California Sur) }\end{array}$ & $1990-2005$ & 18,705 & 17,057 & 0.5 \\
\hline Huizache-Caimanero (Sinaloa) ${ }^{2}$ & 1973-1997 & 2,310 & 1,250 & 2.53 \\
\hline Teacapán-Agua Brava (Sinaloa-Nayarit) ${ }^{3}$ & $1990-2000$ & 89,183 & 75,042 & 1.48 \\
\hline San Blas (Nayarit) $)^{4}$ & $1973-1993$ & 7,644 & 7,379 & 0.2 \\
\hline Barra de Navidad (Jalisco) ${ }^{5}$ & $1985-2000$ & 1,562 & 960 & 3.2 \\
\hline Lagunas de Chacahua (Oaxaca) $^{6}$ & 1986-2007 & 3,014 & 2,998 & 0.02 \\
\hline Soconusco (Chiapas) ${ }^{7}$ & 1994-2011 & 3,118 & 2,609 & 1.05 \\
\hline
\end{tabular}

Fuente: Acosta-Velázquez y Ruíz-Luna (2007); ${ }^{2}$ Ruíz-Luna y Berlanga-Robles (1999); ${ }^{3}$ Acosta-Velázquez (2003); ${ }^{4}$ Berlanga-Robles y Ruíz-Luna (2006); ${ }^{5}$ Holland et al. (2011), ${ }^{6}$ Carbajal-Evaristo (2010); ${ }^{7}$

Con respecto a los valores de forma y dimensión fractal, estos no variaron entre ambas fechas y para el caso de la dimensión fractal, su valor se mantuvo constante. Krummel et al. (1987) reportan que la dimensión 
Emilio I. Romero-Berny, Joanna Acosta-Velázquez, Cristian Tovilla-Hernández,

Birgit Schmook, Rocio Gómez-Ortega. Land coverage changes and fragmentation of

mangroves in the Soconusco Region, Chiapas, Mexico, 1994-2011

fractal disminuye en parches con menor área; mientras que Hargis et al. (1998), señalan que si la fragmentación se manifiesta a manera de parches relativamente homogéneos, los valores de dimensión fractal se mantendrán constantes o con variaciones mínimas aun cuando la fragmentación aumente significativamente. La forma y dimensión fractal, se consideran los mejores indicadores del disturbio humano en un ecosistema, bajo el supuesto de que los parches naturales de vegetación tienden a presentar formas más complejas que los de origen antropogénico (Krummel et al., 1987).

En Ixcuintla-Tecuala, Nayarit, se obtuvo un valor de dimensión fractal de 1.5 (Ramírez, 2006), mayor al estimado en Teacapán Agua BravaMarismas Nacionales de 1.1, el cuál fue considerado como un sistema altamente impactado (Acosta-Velásquez, 2003). El valor obtenido en éste trabajo, permite interpretar que los parches han mantenido formas complejas con cierta tendencia a la regularidad, probablemente, indicando un nivel de impacto antropogénico intermedio.

La exploración visual de los mapas temáticos permite identificar parches grandes, presentándose el mayor en la zona de El Gancho-Murillo que, de acuerdo con este estudio, tuvo una extensión de 1,357.2 ha en 1994 , y se redujo a $1,035.99$ en 2011 , por ende constituye cerca del $40 \%$ de la cobertura total de manglar. Los parches grandes de vegetación natural en un paisaje, generalmente, funcionan como parches parentales y bancos semilleros, por lo que son de especial importancia para la conservación (Acosta, 2003). Es importante tomar estos procesos en el diseño de estrategias de manejo, considerando que la fragmentación conlleva a una reducción en el número de individuos que habitan en un parche, generando poblaciones pequeñas más susceptibles a una extinción estocástica (Krauss et al., 2010).

El análisis de cambios, focaliza afectaciones al manglar por pérdida de cobertura y fragmentación, como ocurre al norte de la laguna El Cabildo, la cuenca baja del río Cahoacán y la zona de El Gancho. En los recorridos de campo se identificaron como agentes causales a una frontera agrícola creciente, principalmente, en la cuenca baja del río Suchiate, la extracción forestal con una tala selectiva de árboles de L. racemosa y Conocarpus erectus, establecimiento de tarquinas o zonas de depósito de sedimentos, así como la presencia de extensos rodales de manglar muerto o en franca regeneración. Tovilla-Hernández et al. (2004), reportan alteraciones de la dinámica natural 
del río Cahoacán, que ha visto disminuido su caudal a causa del problema deforestación-azolvamiento de la cuenca alta, provocando el desplazamiento de su cauce, obstrucción de la bocana del sistema Pozuelos-Murillo y mortalidad de extensas áreas de manglar. Otra problemática asociada a la mortalidad extensiva de manglar, ocurre de manera indirecta por el desazolve de cuerpos de agua para favorecer la pesca ribereña, estableciendo tarquinas directamente en zonas de manglar. También, se detectaron áreas de manglares muertos, con características espectrales similares a las descritas por Acosta-Velázquez (2003). Estas zonas se pueden asociar a la tormenta tropical "Bárbara" (junio 2007), que impactó Puerto Chiapas, ocasionando la destrucción de manglares, principalmente, en las zonas cercanas a El Gancho-Murillo. En general, el impacto de los huracanes en los manglares es un fenómeno poco estudiado en México a pesar de ser eventos frecuentes en los litorales, tanto del Pacífico como del Atlántico.

Tovilla-Hernández y Romero-Berny (2012), estiman que un 26\% del manglar en el sistema El Cabildo-Amatal se ha visto afectado por la tala. El plan de manejo de las áreas protegidas estatales Cabildo-Amatal y Gancho-Murillo, presenta un mapa de zonificación de las reservas incluyendo zona de uso restringido, zona de recuperación y zona de aprovechamiento sustentable, indicando que en todas la extracción de madera de mangle se encuentra prohibida en cumplimiento de la NOM-ECOL-059 (Gobierno de Chiapas 2010a; 2010b). No obstante, el impacto de las comunidades humanas al interior de las reservas es evidente, siendo común el uso de madera de mangle. En algunas localidades de Gancho-Murillo, como Barra de Cahoacán, Conquista Campesina y La Cigüeña, se cuenta con trabajos de manejo y conservación del manglar (Tovilla-Hernández et al., 2004; Rojas-Garcias, 2012), aunque de manera general, el desconocimiento de la regulación establecida en los programas de manejo es extendido.

\section{Conclusiones}

De manera, general puede concluirse que la generación de cartografía por interpretación y segmentación, permite cuantificar con alta exactitud la cobertura de manglar y su dinámica a niveles de clase y paisaje. El análisis permitió estimar la extensión del manglar en 1994 en 3,118 ha; y en 2011 en 2,609 ha, calculando éste último dato con un valor de exactitud elevado. Se estimó una pérdida neta de 509 ha de manglar, a una tasa de deforestación 
Emilio I. Romero-Berny, Joanna Acosta-Velázquez, Cristian Tovilla-Hernández,

Birgit Schmook, Rocio Gómez-Ortega. Land coverage changes and fragmentation of

mangroves in the Soconusco Region, Chiapas, Mexico, 1994-2011

anual de $1.05 \%$ para un periodo de 17 años, siendo un valor alto para áreas sujetas a conservación. La vegetación de manglar está sujeta a un proceso de fragmentación a una tasa de $4.9 \%$, sin embargo el $40 \%$ de la cobertura total es constituida por un parche continuo. Asimismo, las métricas de forma indican cierta complejidad en los parches, por lo que se considera un nivel de impacto antropogénico intermedio a nivel de la clase.

Dada la dinámica de paisaje en el Soconusco, es urgente estudiar a mayor detalle los procesos espaciales del manglar en relación con otros usos del suelo. Asimismo es necesario contar con información referente a las comunidades bióticas que utilizan como hábitat a los parches a fin de comprender como la fragmentación impacta sobre la conectividad del sistema. Es altamente recomendable, continuar con los programas de restauración y rehabilitación del manglar, así como un manejo integrado de las cuencas de los ríos Coatán, Cahoacán y Suchiate, a fin de reducir la vulnerabilidad de la zona ante fenómenos hidrometeorológicos. Finalmente, es de primordial importancia promover el desarrollo de las localidades asentadas en el área, bajo un enfoque de sustentabilidad que favorezca el co-manejo de las áreas naturales protegidas.

\section{Referencias}

Acosta-Velásquez, J. (2003). Análisis de la condición del bosque de manglar en el sistema lagunar Teacapán-Agua Brava-Marismas Nacionales y sus relaciones a nivel de paisaje. Tesis de Maestría. Centro de Investigación en Alimentación y Desarrollo. México, 89.

Acosta-Velázquez, J., y Ruíz-Luna, A. (2007). "Variación en la cobertura, distribución y estructura de los manglares del complejo lagunar Bahía Magdalena-Bahía Almejas (1990-2005)”. Estudios ecológicos en Bahía Magdalena. La Paz, Baja California Sur, México: FunesRodríguez, R., Gómez-Gutiérrez, J. y Palomares-García, R. (Editores), CICIMAR-IPN. 127-141.

Agraz-Hernández, C. M., y Flores-Verdugo, F. J. (2005). "Diagnóstico del impacto y lineamientos básicos para los programas de mitigación y manejo de humedales". Golfo de México, contaminación e impacto ambiental: diagnóstico y tendencias. Campeche, Campeche, México: Botello, A. V., Rendón-Von Osten, J., Gold-Bouchot, G., y Agraz-Hernández, C. M. (Editores), EPOMEX-UAC/UNAM/INE/UJAT/UIA. 597-606. 
Altamirano, A., Miranda, A. y Jiménez, C. (2012). Incertidumbre de los índices de paisaje en el análisis de la estructura espacial. Bosque, 33 (2), 171-181.

Arriaga-Cabrera, L., Vásquez-Domínguez, E., González-Cano, J., JiménezRosenberg, R., Muñoz-López, E. y Aguilar-Sierra, V. (1998). Regiones Hidrológicas Prioritarias de México. Comisión Nacional para el Conocimiento y uso de la Biodiversidad. Recuperado de www.conabio.gob.mx

Bassols, B.A., Rodríguez, D.G., Vargas, G.B., Sandoval L., y Ortiz, A. (1974). La costa de Chiapas: un estudio económico regional. Distrito Federal, México: UNAM-IIE.

Berlanga-Robles, C.A., y Ruíz-Luna, A. (2006). Evaluación de cambios en el paisaje y sus efectos sobre los humedales costeros del sistema estuarino de San Blas, Nayarit (México) por medio del análisis de imágenes LANDSAT. Ciencias Marinas, 32(3), 523-538.

Carbajal, E.S.S. (2010). Estudio de la estructura, composición y modificación antrópica de los manglares del Parque Nacional Lagunas de Chacahua, Oaxaca. Tesis de Licenciatura. Universidad Autónoma de Guerrero, México, 112.

CONABIO. (2009). Manglares de México. Comisión Nacional para el Conocimiento y Uso de la Biodiversidad. Recuperado de www. biodiversidad.gob.mx/ecosistemas/manglares

Congalton, R.G., y Green, K. (1999). Assessing the Accuracy of Remotely Sensed Data: Principles and Practice. Boca Raton, Florida, EUA: Lewis Publishers.

Díaz-Gallegos, J.R., y Acosta-Velázquez, J. (2009). “Tendencias en la transformación del uso de suelo y la vegetación aledaña". El Sistema Ecológico de la Bahía Chetumal/Corozal: Costa occidental del Mar Caribe. Chetumal, Quintana Roo, México: Espinoza-Avalos, J., Islebe, G.A., y Hernández-Arana, H.A. (Editores), ECOSUR. 226-237. Duke, N., Meynecke, J.O., Dittman, S., Ellison, A.M., Anger, K., Berger, U., Cannici, S., Diele, K., Ewel, K.C., Field, C.D., Koedam, N., Lee, S.Y., Marchand, C., Nordhaus, I. y Dahdouh-Guebas, F. (2007). A world without mangroves?. Science, 317, 41-42.

Elkie, P.C., Rempel, R.S. y Carr, A.P. (1999). Patch Analyst User's Manual: A tool to quantifying landscape structure. Manual 002. Ontario, Canadá: NWST. 
Emilio I. Romero-Berny, Joanna Acosta-Velázquez, Cristian Tovilla-Hernández,

Birgit Schmook, Rocio Gómez-Ortega. Land coverage changes and fragmentation of

mangroves in the Soconusco Region, Chiapas, Mexico, 1994-2011

FAO. (1996). Forest resources assessment. Forestry paper, 130. Roma, Italia: Food and Agriculture Organization of the United Nations.

FAO. (2007). The world's mangroves 1980-2005. Forestry paper, 153. Roma, Italia: Food and Agriculture Organization of the United Nations.

Flores-Verdugo, F. J., Agraz-Hernández, C. M. y Benítez-Pardo, D. (2007).

"Ecosistemas acuáticos costeros: importancia, retos y prioridades para su conservación". Perspectivas sobre conservación de ecosistemas acuáticos en México: Distrito Federal, México: Sánchez, O., Herzig, M., Peters, E., Márquez, R. y Zambrano, L. (Editores), INE-SEMARNAT. 147-166.

Giri, C., Ochieng, E., Tieszen, L., Zhu, Z., Singh, A., Loveland, T., Masek, J. y Duke, N. (2011). Status and distribution of mangrove forests of the world using earth observation satellite data. Global Ecology and Biogeography, 20, 154-159.

Gobierno de Chiapas. (2010b). Programa de Manejo de la Zona Sujeta a Conservación Ecológica El Gancho-Murillo: Tuxtla Gutiérrez, Chiapas, México. México: Secretaría de Medio Ambiente y Vivienda.

Hargis, C.D., Bissonette, J.A. y David, J.L. (1998). The behavior of landscape metrics commonly used in the study of habitat fragmentation. Landscape Ecology, 13(3), 167-183.

Holland, T.L., Mariscal, R.J., Davidson, A.R. y Cardillé, J. (2011). Landscape changes in a coastal system undergoing tourism development: implications for Barra de Navidad Lagoon, Jalisco, Mexico. Investigaciones Geográficas, 74, 7-18.

Jones, H.G. y Vaughan, R.A. (2010). Remote sensing of Vegetation. Principles, Techniques and Applications. Nueva York, EUA: Oxford University Press.

Krauss, J., Bommarco, R., Guardiola, M., Heikkinen, R.K., Helm, A., Kuussaari, M., Lindborg, R., Ockinger, E., Partel, M., Pino, J., Poyry, J., Raatikainen, K.M., Sang, A., Stefanescu, C., Teder, T., Zobel, M. y Steffan-Dewenter, I. (2010). Habitat fragmentation causes immediate and time-delayed biodiversity loss at different trophic levels. Ecology Letters, 13, 597-605.

Krummel, J.R., Gardner, R.H., Sugihara, G., O'Neill, R.V. y Coleman, P.R. (1987). Landscape patterns in a disturbed environment. Oikos, 48(3), 321-324. 
McGarigal, K. (2002). Landscape pattern metrics. Universidad de Massachusetts. Recuperado de www.umass.edu/landeco/pubs/pubs

Monzalvo, S.I.K. (2006). Estimación de la cobertura espacial y análisis de la estructura forestal del manglar en Sinaloa, aplicando técnicas de percepción remota. Tesis de Maestría. Instituto de Ciencias del Mar y Limnología, Universidad Nacional Autónoma de México. México. 84.

Ovalle-Estrada, F. y Vásquez-Lule, A. D. (2009). Caracterización del sitio de manglar Lagunas Cabildo - Amatal-Gancho Murillo. Comisión Nacional para el Conocimiento y Uso de la Biodiversidad. Recuperado de www. biodiversidad.gob.mx/ecosistemas/manglares

Ramírez, B. P. R. (2006). Análisis espacial de la cobertura de manglar en los municipios de Tecuala y Santiago Ixcuintla, Nayarit en el periodo 1973-2005. Tesis de Maestría. Centro de Investigación en Alimentación y Desarrollo. México, 95.

Rojas-Garcias, J. (2012). Community strategy for mangrove forest conservation: Conquista Campesina Conservation Easement. Field Action Science Reports, 7. Recuperado de http://factreports.revues.org/2197

Ruíz-Luna, A. y Berlanga-Robles, C.A. (1999). Modifications in coverage patterns and land use in the landscape around the Huizache-Caimanero lagoon system, Sinaloa, México: a multitemporal analysis LANDSAT images. Estuarine, Coastal and Shelf Science, 49, 37-44.

Sánchez, J. E. y Járquin, R. (2004). La Frontera Sur. Reflexiones sobre el Soconusco, Chiapas y sus problemas ambientales, poblacionales y productivos. México: Senado de la Republica LX Legislatura, ECOSUR.

Tovilla-Hernández, C. y Romero-Berny, E. I. (2012). "Diagnóstico estructural de los manglares de Chiapas y Oaxaca". Recursos acuáticos costeros del Sureste, Volumen 1. Mérida, Yucatán, México: Sánchez, A.J., Chiappa-Carrará, X. y Brito, P. R. (Editores), CONACYT, RECORECOS, UNAM, 257-279.

Tovilla-Hernández, C., Román-Salazar, A.V., Simuta-Morales, G.M. y Linares-Mazariegos, R. M. (2004). Recuperación del manglar en la Barra del río Cahoacán en la costa de Chiapas. Madera y Bosques, 10(2), 77-91.

Valiela, I., Bowen, J. L. y York, J. K. (2001). Mangrove forests: one of the world's threatened major tropical environments. BioScience, 51(10), 807-815. 